\title{
Explanation of Giant Cluster Coexistence In Doped Manganites and Other Compounds
}

\author{
Adriana Moreo, Matthias Mayr, Adrian Feiguin, Seiji Yunoki, and Elbio Dagotto \\ National High Magnetic Field Lab and Department of Physics, \\ Florida State University, Tallahassee, FL 32306, USA
}

(Submitted 12 November 1999)

\begin{abstract}
Computational studies show the generation of large coexisting metallic and insulating clusters with equal electronic density in models for manganites. The clusters are induced by disorder on exchange and hopping amplitudes near first-order transitions of the nondisordered strongly coupled system. The random-field Ising model is used to explain the qualitative aspects of our results. Percolative characteristics are natural in this context. Our results explain the recently experimentally discovered micrometer size inhomogeneities in manganites. The conclusions are general and apply to a variety of compounds.
\end{abstract}

Considerable work is currently being focussed on the experimental and theoretical study of manganese oxides. This huge effort was triggered by the discovery of Colossal Magnetoresistance (CMR) in manganites (1), where the resistivity changes by several orders of magnitude upon the application of modest fields of a few Tesla at the carrier densities and temperatures where metallic and insulating phases are in competition. Theoretical investigations of simple manganite models based upon numerical simulations and mean-field approximations have reproduced some of the complex spin-, orbital- and charge-ordered phases observed experimentally. In particular, the charge-ordered (CO) CE-state of halfdoped manganites has been recently stabilized in Monte Carlo (MC) simulations of the two-orbital model with Jahn-Teller phonons (2). This state is in competition with the ferromagnetic $(\mathrm{FM})$ and A-type states also observed in experiments at the hole density $\mathrm{x}=0.5$. However, the curious magnetotransport properties of manganites has resisted theoretical understanding and a proper explanation of the CMR phenomenon is still lacking. 
Recently, important new experimental information about the microscopic properties of manganites has been reported. Using electronic diffraction and transport techniques, Uehara et al. have investigated the effect of Pr-doping upon the metallic ferromagnetic compound $\mathrm{La}_{5 / 8} \mathrm{Ca}_{3 / 8} \mathrm{MnO}_{3}$ (3). As the Pr density (y) increases by the replacement $\mathrm{La} \rightarrow \operatorname{Pr}$, the system changes at $\mathrm{y} \sim 0.35$ to an insulating CO-state. At low temperature in this regime the unexpected coexistence of giant clusters of FM and CO phases was observed (3). Similar results were reported by Fäth et al. using scanning tunneling spectroscopy applied to $\mathrm{La}_{0.7} \mathrm{Ca}_{0.3} \mathrm{MnO}_{3}$ (4). At temperatures close to the Curie temperature, thinfilm spectroscopic images revealed a complicated pattern of interpenetrating giant metallic and insulating phases. The clusters found in both these experiments were as large as 0.1 $\mu \mathrm{m}=1000 \AA \sim 250 a$, with $a \sim 4 \AA$ the Mn-Mn distance $(3,4)$. The metal-insulator FM-CO transition occurs through a percolative process among the clusters, as a function of either temperature or magnetic field. These results rule out the picture of homogeneously distributed small polarons to describe doped manganites in the CMR regime.

The discovery of huge coexisting FM-CO clusters in a manganite single-crystal is puzzling. The only theoretical framework which in principle could be used to address this issue is the phase separation (PS) scenario where mixed-phase characteristics, involving phases with different electronic densities, are natural (5). The PS ideas are indeed successful in describing manganites at, e.g., small hole density, where nanometer size inhomogeneities have been widely discussed (5), and at high densities $\mathrm{x} \sim 1$ based on recent magnetic and transport data (6). However, the micrometer clusters at intermediate densities found in Refs. $(3,4)$ appear to require an alternative explanation since the energy cost of charged $\mu \mathrm{m}$-size domains would be too large to keep the structure stable. Actually, explicit numerical calculations in one dimensional (1D) models have shown that the large clusters in PS regimes break down into smaller pieces of a few lattice spacings in size upon the introduction of a nearest-neighbor charge repulsion (7). In addition, this repulsion tends to arrange the charge in an ordered pattern (7) -charge-density-waves or stripes- contrary to the random location and shape of the clusters observed experimentally $(3,4)$. A novel framework involving large clusters with equal-density phases is needed to rationalize the results of Refs. $(3,4)$. 
In the absence of theoretical proposals to explain the giant FM-CO clusters it is necessary to reconsider some of the properties of the models studied thus far. Of particular relevance is the assumption of translationally invariant interactions, which is at odds with the chaotic looking appearance of microclusters in experiments $(3,4)$. For this reason, here we report a computational study of manganite models which combines (i) strong coupling interactions, necessary to reproduce the rich variety of ordered phases of these materials, and (ii) quenched disorder. The latter is caused by the random chemical replacement of ions, such as La and Pr, with different ionic sizes. This replacement affects the hopping amplitudes of $\mathrm{e}_{\mathrm{g}}$-electrons due to the buckling of the Mn-O-Mn bonds near Pr (8). Recent calculations showed that the concomitant modification of the exchange coupling $\mathrm{J}_{\mathrm{AF}}$ among the $t_{2 g}$-spins is likely equally important in establishing the properties of manganite models (2). Thus, by considering randomly chosen hopping and exchange couplings fluctuating about the non-disordered values of interest, the physics of doped manganites will be more properly captured. Following this procedure, here we report the natural appearance of coexisting giant clusters of equal-density FM and AF phases in realistic models. The conclusions are general and similar cluster formation is expected for a variety of compounds.

To present our main results first consider the two-orbital model, described extensively in previous work $(5,9)$. It contains (i) an electronic hopping term, regulated by amplitudes $\mathrm{t}_{\mathrm{ab}}^{\alpha}$, with a,b=1,2 labeling the $\mathrm{d}_{\mathrm{x}^{2}-\mathrm{y}^{2}}$ and $\mathrm{d}_{3 \mathrm{z}^{2}-\mathrm{r}^{2}}$ orbitals, $\alpha=\mathrm{x}, \mathrm{y}, \mathrm{z}$ being the axes directions, and $t_{11}^{\mathrm{x}}=\mathrm{t}$ the energy scale, (ii) a strong FM coupling between the localized $\mathrm{t}_{2 \mathrm{~g}}$ - and mobile e -fermions, regulated by $\mathrm{J}_{\mathrm{H}}$, (iii) a direct antiferromagnetic (AF) exchange among the localized spins with strength $\mathrm{J}_{\mathrm{AF}}$, and (iv) an electron-phonon coupling between the $\mathrm{Q}_{2}$ and $\mathrm{Q}_{3}$ Jahn-Teller modes and the mobile electrons, with strength $\lambda$. The phase diagram of the non-disordered model was studied by standard MC simulations using classical localized spins and phonons $(2,9)$. Similar results were obtained with mean-field approximations including Coulombic repulsions (2). The generality and rationalization of the numerical data described below suggest that the main conclusions are actually independent of the detailed properties of the competing states. Whether the phases are generated by phononic, magnetic, or Coulombic interactions appears unimportant.

The focus of our studies will be on first-order transitions, which in the two-orbital model occur in several locations in parameter space in any dimension of interest $(2,5,9)$. 
However, for the disorder-induced cluster formation described below it is more convenient to analyze 1D systems first since the two-orbital 2D lattices that can be studied computationally are not sufficiently large. Among the possible 1D first-order transitions, results are here reported for the transition occurring between FM and AF states at fixed $\mathrm{x}=0.5$ and large $\lambda$, as a function of $\mathrm{J}_{\mathrm{AF}} / \mathrm{t}$. The AF phase studied has a four-spin unit cell $\uparrow \uparrow \downarrow \downarrow$, and a concomitant peak in the spin structure factor $\mathrm{S}(\mathrm{q})$ at $\mathrm{q}=\pi / 2(2,10)$. The state is insulating, as demonstrated by the absence of Drude weight and the vanishing density of states at the Fermi energy. Nearest-neighbors correlations among the $t_{2 g}$-spins are used to distinguish among the FM and AF phases. In Fig.1A the energy per site (E) vs $\mathrm{J}_{\mathrm{AF}} / \mathrm{t}$ for the non-disordered model is shown. The $\mathrm{dE} / \mathrm{d}\left(\mathrm{J}_{\mathrm{AF}} / \mathrm{t}\right)$ discontinuity indicates the first-order character of the transition at $\mathrm{J}_{\mathrm{AF}} /\left.\mathrm{t}\right|_{\mathrm{c}} \approx 0.21$. Disorder is introduced in $\mathrm{t}_{\mathrm{ab}}^{\alpha}$ and $\mathrm{J}_{\mathrm{AF}}$ such that $\mathrm{J}_{\mathrm{AF}} / \mathrm{t}$ becomes effectively random in the interval $\mathrm{J}_{\mathrm{AF}} /\left.\mathrm{t}\right|_{\mathrm{c}^{-}} \delta$ to $\mathrm{J}_{\mathrm{AF}} /\left.\mathrm{t}\right|_{\mathrm{c}}+\delta$. Results for one fixed set of couplings are shown for $\delta=0.01$ in Fig.1B (other sets lead to similar results). The MC averaged correlations in Fig.1B already show one of the main results of this paper, namely the remarkable formation of coexisting large FM and AF clusters in the ground state, typically of order $10 a$ each ( $a$ is the lattice spacing). This occurs even though $\mathrm{J}_{\mathrm{AF}} / \mathrm{t}$ at each link (not shown) rapidly changes at the $a$ scale since different sites are uncorrelated in the disorder. Naively it may have been expected that at every link either the FM or AF phases would be stable depending on the value of $\mathrm{J}_{\mathrm{AF}} / \mathrm{t}$, as it occurs for a dominant strong disorder. However, at weak disorder this would produce a large interface energy and the order parameter cannot follow the rapid oscillations of $\mathrm{J}_{\mathrm{AF}} / \mathrm{t}$ from site to site. As a consequence, structures much larger than the lattice spacing emerge, with a size regulated by $\delta$ (for instance, in Fig.1C results at $\delta=0.05$ contain FM clusters smaller than in Fig.1B). The effect occurs only near first-order transitions, i.e. the same weak disorder in other regions does not produce important effects in the spin correlations. Qualitatively similar results appear also in other first-order transitions of the two-orbital model, such as for the FM-CO(CE-state) level crossing reported in Ref.(2) using $4 \times 4$ and $4 \times 4 \times 2$ clusters. The generation of large equal-density clusters by $\left(t, \mathrm{~J}_{\mathrm{AF}}\right)$-disorder near first-order transitions is an effect unforeseen in previous manganite investigations.

The rapid CPU time growth with cluster size of the two-orbital model does not allow us to investigate numerically the phenomenon in more detail than shown in Figs.1A-C. 
Fortunately, there are simpler models with the same behavior, including the well-known one-orbital model (5). It contains hopping for only one species of $\mathrm{e}_{\mathrm{g}}$-electrons (regulated by $\mathrm{t}$ ), a FM Hund coupling $\mathrm{J}_{\mathrm{H}}$ linking the $\mathrm{e}_{\mathrm{g}}$ - and (classical) $\mathrm{t}_{2 \mathrm{~g}}$-spins, and a direct exchange $\mathrm{J}_{\mathrm{AF}}$ among the $\mathrm{t}_{2 \mathrm{~g}}$-spins. Previous work showed that this model also has a first-order transition at $\mathrm{x}=0.5$ as $\mathrm{J}_{\mathrm{AF}} / \mathrm{t}$ varies, in the large $\mathrm{J}_{\mathrm{H}}$ regime (10). It involves equal-density metallic FM and insulating AF states, the latter with a similar spin structure as the AF state of Figs.1A-C. To investigate disorder effects here the natural modification is to select the exchange $\mathrm{J}_{\mathrm{AF}}$ randomly in the interval $\left[\mathrm{J}_{\mathrm{AF}}^{\mathrm{c}}-\delta, \mathrm{J}_{\mathrm{AF}}^{\mathrm{c}}+\delta\right](11)$, where $\mathrm{J}_{\mathrm{AF}}^{\mathrm{c}} \sim 0.14$ is the critical first-order transition coupling at $\mathrm{J}_{\mathrm{H}}=\infty, \mathrm{t}=1$, and $\mathrm{T}=1 / 70$ in the non-disordered limit (Fig.1D). In Fig.1E, results of a MC simulation corresponding to a representative set of random couplings $\left(\mathrm{t}, \mathrm{J}_{\mathrm{AF}}\right)$ centered at 0.14 are shown. As in the two-orbital case, FM and AF clusters, this time as large as 20a, are easily obtained. S(q) (not shown) contains a double-peak structure with dominant features indicating a FM-AF mixed phase. If the range of possible $\left(\mathrm{t}, \mathrm{J}_{\mathrm{AF}}\right)$ increases, the cluster size decreases (Fig.1F). Open boundary conditions (OBC) were used in Figs.1D-F, and periodic boundary conditions (PBC) in Figs.1A-C, to show that the large cluster formation occurs independently of these details.

To further investigate the universality of the large cluster generation phenomenon, note that the non-disordered one-orbital model has another prominent first-order transition corresponding to a discontinuity in the density $\langle\mathrm{n}\rangle$ vs chemical potential $\mu$, for a wide range of couplings (5). The direct interpretation of such a result is the presence of PS between competing FM and AF states (5). However, in the context emphasized here the focus shifts from the transition properties to the effect of disorder on the $\langle\mathrm{n}\rangle$ discontinuity itself. Disorder is here naturally introduced as a site-dependent chemical potential of the form $\sum_{\mathbf{i}} \phi_{\mathbf{i}} \mathrm{n}_{\mathbf{i}}$, where $\phi_{\mathbf{i}}$ is randomly selected in the interval $\left[-\frac{\mathrm{W}}{2},+\frac{\mathrm{W}}{2}\right]$, and $\mathrm{n}_{\mathbf{i}}$ is the electronic number operator at site $\mathbf{i}$. Results of a standard $\mathrm{MC}$ simulation for a $\mathrm{L}=20$ sites chain of the disordered one-orbital model are in Fig.2A. Averages of $\langle\mathrm{n}\rangle$ over 100 disorder configurations are shown. For the values of $\mathrm{W}$ studied here, $\langle\mathrm{n}\rangle$ no longer has a discontinuity. For small $\mathrm{W}$ the first-order transition is replaced by a rapid crossover, where the compressibility proportional to $d \mu / d\langle n\rangle$ remains high, suggesting the formation of large clusters. This is confirmed in Fig.2B where the nearest-neighbor $t_{2 \mathrm{~g}}$-spin correlations are shown for a $\mathrm{L}=60$ chain, two disorder configurations, and one (typical) MC snapshot for each. These results 
are very similar if averages over the MC configurations are made, showing that the system is basically frozen into an inhomogeneous ground state with large AF and FM clusters of size $\sim 10-20 a$, similarly as in Fig.1B,E. Once again, reducing $\mathrm{W}$ increases the cluster sizes, only limited by the size of the systems that can be studied computationally. Here it was also observed that the mixed-phase ground state leads to a pseudogap in the $\mathrm{T} \sim 0$ density of states (Fig.2C), as it occurs in non-disordered models at finite temperatures in particular regions of parameter space (12). An analogous pseudogap was also observed working with the two-orbital model. Similar results as in Figs.2A-C were also found in two dimensions (2D) (see, e.g., Fig.2D) and thus the large cluster formation certainly does not depend on pathological properties of $1 \mathrm{D}$ systems. Note that in the particular example studied in Figs.2A-D, the AF-FM regions involved have different electronic densities, complementing the results of Figs.1A-F with equal-density clusters. In both cases the results are illustrative of cluster formation induced by disorder.

The simplicity and universality of the MC simulation results with large coexisting FMAF clusters suggest that there is a general principle at work in the problem. To understand this effect let us briefly review the phenomenology of the random-field Ising model (RFIM) (13) defined by the Hamiltonian $H=-J \sum_{\langle\mathbf{i} \mathbf{j}\rangle} S_{\mathbf{i}} S_{\mathbf{j}}-\sum_{\mathbf{i}} h_{\mathbf{i}} S_{\mathbf{i}}$, where $S_{\mathbf{i}}= \pm 1$, and the rest of the notation is standard. The random fields $\left\{h_{\mathbf{i}}\right\}$ have the properties $\left[h_{\mathbf{i}}\right]_{\mathrm{av}}=0$ and $\left[\mathrm{h}_{\mathbf{i}}^{2}\right]_{\mathrm{av}}=\mathrm{h}^{2}$, where $\mathrm{h}$ characterizes the width of the distribution, and $[\ldots]_{\mathrm{av}}$ is the average over the fields. In manganites the Ising variables represent the competing metallic and insulating states on a small region of space centered at $\mathbf{i}$. The random field mimics the $t_{\mathrm{ab}}^{\alpha}$ and $\mathrm{J}_{\mathrm{AF}}$ fluctuations locally favoring one state over the other. Without disorder, the Ising model has a first-order transition at zero magnetic field and $\mathrm{T}=0$ between the two fullyordered states, analogous to the AF-FM first-order transitions of non-disordered manganite models (2). However, at $\mathrm{h} \neq 0$ the properties of the Ising transition are drastically affected (13). The key arguments guiding RFIM investigations (14) can be restated for manganites. Working very close to a first-order transition, consider that in a region dominated by phase-I (either AF or FM), a phase-II bubble of radius $\mathrm{R}$ is created. The energy cost $\mathrm{R}^{\mathrm{d}-1}$ is proportional to the domain wall area, with $d$ the spatial dimension. To stabilize the bubble it is necessary to induce an energy compensation originated in the $\left(t, \mathrm{~J}_{\mathrm{AF}}\right)$ disorder. Consider the average hopping inside the bubble using $S_{R}=\sum_{l} t_{l}$, where 1 labels bonds and $t_{1}$ 
is the hopping deviation at bond 1 from its non-disordered value, the latter of which is fixed at the critical coupling of the first-order transition of the non-disordered model. Although the random hopping deviations mostly cancel inside the bubble, important fluctuations must be considered. In particular, the standard deviation of $S_{R}$ is $\sigma_{S_{R}}=(\Delta t) R^{d / 2}$ since $\left[\mathrm{t}_{1} \mathrm{t}_{\mathrm{l}^{\prime}}\right]_{\mathrm{av}}=(\Delta \mathrm{t})^{2} \delta_{\mathrm{ll}^{\prime}}$, with $(\Delta \mathrm{t})$ characterizing the width of the random hopping distribution about the non-disordered value. A similar expression holds for the $\mathrm{J}_{\mathrm{AF}}$ fluctuations. Then, centered at any lattice site it is always possible to find a region of size $\mathrm{R}$, such that at least the average couplings favor either phase-I or -II with a substantial strength of order $\mathrm{R}^{\mathrm{d} / 2}$, although individual random deviations $\mathrm{t}_{1}$ cannot exceed a (small number) $\Delta \mathrm{t}$.

To illustrate the generation of large clusters in the RFIM, standard MC simulations were performed. Although similar MC studies have been discussed in the RFIM framework (13), the results shown here provide useful qualitative information to manganite experts. In Fig.3A, low temperature results are shown for one representative set $\left\{h_{\mathbf{i}}\right\}$ individually taken from $[-\mathrm{W},+\mathrm{W}]$ with $\mathrm{W}=3.0$, in units of $\mathrm{J}=1(\mathrm{~W}=\sqrt{3} \mathrm{~h})$. The dynamical formation of large coexisting clusters is clear, in spite of the uncorrelated character of the random fields in neighboring sites. Using the same set $\left\{\mathrm{h}_{\mathbf{i}}\right\}$ as in Fig.3A but rescaling its intensity with W, Fig.3B shows that as $\mathrm{W}$ is reduced the typical cluster sizes rapidly grow and at $\mathrm{W}=1.5$ clusters as large as $50 \mathrm{a}$ in characteristic length are possible. Fig.3C contains simulation results now on a large $500 \times 500$ lattice showing that RFIM cluster sizes can be made as large as those found in manganite experiments (250a) by simply adjusting W. Fig.3D illustrates the influence of an external field $-\mathrm{H}_{\text {ext }} \sum_{\mathrm{i}} \mathrm{S}_{\mathbf{i}}$ added to the Hamiltonian. As $\mathrm{H}_{\text {ext }}$ grows the region most affected by the field is the surface of the spin down domains, which are transformed into spin up. This tends to suppress the narrowest regions of the spin down clusters, as highlighted with arrows in Fig.3D, providing a field-induced connection among spin up regions that otherwise would be disconnected. Then, intuitively, as $\mathrm{H}_{\mathrm{ext}}$ increases a percolative transition is to be expected. Based on the RFIM-manganite analogy, the picture described here predicts a similar percolative transition involving metallic and insulating clusters as chemical compositions, temperatures, or magnetic fields are varied near first-order transitions, as observed experimentally $(3,4)$. Giant cluster generation by weak disorder in manganite models and in the RFIM appear related phenomena. However, at this early stage in the calculations it is difficult to predict critical exponents for 
the metal-insulator transition. Even for simpler spin systems such as diluted anisotropic antiferromagnets there is still no full agreement between RFIM theory and experiments (13). In addition, the manganite critical dimension may be affected by the 1D character of the zig-zag chains that form the planar CE-state (15), and critical slowing down as in the RFIM can produce rounding effects that make a comparison between scaling theory and manganite experiments difficult.

The ideas described here are not limited to particular manganite compounds but they apply to other materials where a transition with first-order characteristics occurs, either by varying temperatures in compounds with some source of disorder, or by explicit chemical substitution which leads to quenched fluctuations in the hopping and exchange amplitudes. For instance, other manganites such as $\left(\mathrm{La}_{1-\mathrm{x}} \mathrm{Tb}_{\mathrm{x}}\right)_{2 / 3} \mathrm{Ca}_{1 / 3} \mathrm{MnO}_{3}$ and $\mathrm{La}_{2-2 \mathrm{x}} \mathrm{Sr}_{1+2 \mathrm{x}} \mathrm{Mn}_{2} \mathrm{O}_{7}$ also have a AF-FM competition at low temperatures. While previous investigations assigned spin-glass (16) or canted-phase (17) characteristics to the intermediate region, mixedphase properties involving equal-density large clusters as found in $\mathrm{La}_{5 / 8-y} \mathrm{Pr}_{\mathrm{y}} \mathrm{Ca}_{3 / 8} \mathrm{MnO}_{3}$ provide an alternative description. In $\mathrm{La}_{0.5} \mathrm{Ca}_{0.5} \mathrm{MnO}_{3}$, large $\mathrm{FM}$ and $\mathrm{CO}$ clusters have also been reported (18), and the influence of disorder on the first-order transition is a possible explanation for their existence (19). The concepts discussed here also apply to ruthenates, such as $\left(\mathrm{Sr}_{1-y} \mathrm{Ca}_{\mathrm{y}}\right)_{3} \mathrm{Ru}_{2} \mathrm{O}_{7}$, where a difficult-to-characterize y 0.5 region separates $\mathrm{FM}$ and $\mathrm{AF}$ phases (20). The metal-insulator transition of $\mathrm{LaNi}_{1-\mathrm{x}} \mathrm{Fe}_{\mathrm{x}} \mathrm{O}_{3}$ (21) may also proceed through a mixed-phase (equal-density) regime with giant cluster formation. In addition, $\mathrm{EuB}_{6}$ behaves similarly to manganites (22) and it may present an analogous percolative behavior caused by disorder. The same could occur for the transition metal chalcogenide $\mathrm{NiS}_{2-\mathrm{x}} \mathrm{Se}_{\mathrm{x}}$ at $\mathrm{x} \sim 0.5$ (23). Cr alloys such as $\mathrm{Cr}_{1-\mathrm{x}} \mathrm{Fe}_{\mathrm{x}}$, may also have an interesting AF-FM competition with percolative properties. Finally, the notorious inhomogeneities observed experimentally in high temperature superconductors, such as $\mathrm{La}_{2-\mathrm{x}} \mathrm{Sr}_{\mathrm{x}} \mathrm{CuO}_{4}$, in regimes of low density of carriers may be caused in part by disordering effects on first-order transitions.

Summarizing, based on the calculations reported here, the giant clusters in manganites found experimentally in Refs. $(3,4)$ are conjectured to be caused by quenched disorder in the couplings ( $\mathrm{t}_{\mathrm{ab}}^{\alpha}$ and $\mathrm{J}_{\mathrm{AF}}$ ) of the system, which are induced by chemical substitution, and which affect transitions that otherwise would be of first-order without disorder (24). 
A pseudogap in the density of states appears in this regime. The mixed-phase state presented in Figs.1A-F involves clusters with equal electronic density, complementing the phase separation scenario which involves regions with different densities (5). In phase-separated regimes disorder also leads to cluster formation (Figs.2A-D). Although non-disordered models remain crucial to determining the competing tendencies in manganites and to establish the order of the phase transitions, disordering effects appear necessary to reproduce the subtle percolative nature of the metal-insulator transition and the conspicuous presence of $\mu \mathrm{m}$ domains in these compounds in regimes near first-order transitions $(3,4)$. The present observations are general, not based on fine tuning of models or parameters, and they should apply to a variety of other compounds as well. The formation of coexisting giant clusters when two states are in competition through first-order transitions should be a phenomenon frequently present in transition-metal-oxides and related compounds.

\section{REFERENCES}

1. Y. Tokura et al., J. Appl. Phys. 79, 5288 (1996); A. P. Ramirez, J. Phys.: Condens. Matter 9, 8171 (1997).

2. S. Yunoki, T. Hotta, and E. Dagotto, preprint, cond-mat/9909254.

3. M. Uehara, S. Mori, C. H. Chen, and S.-W. Cheong, Nature 399, 560 (1999).

4. M. Fäth, S. Freisem, A. A. Menovsky, Y. Tomioka, J. Aarts, and J. A. Mydosh, Science 285, 1540 (1999).

5. A. Moreo, S. Yunoki and E. Dagotto, Science 283, 2034 (1999); and references therein.

6. J. J. Neumeier and J. L. Cohn, preprint.

7. A. L. Malvezzi, S. Yunoki, and E. Dagotto, Phys. Rev. B59, 7033 (1999).

8. S.-W. Cheong, and H. Y. Hwang, in Colossal Magnetoresistance Oxides, ed. Y. Tokura, Monogr. in Condensed Matter Sci., Gordon \& Breach, London, 1999.

9. S. Yunoki, A. Moreo, and E. Dagotto, Phys. Rev. Lett. 81, 5612 (1998). See also A. J. Millis, B. Shraiman and R. Mueller, Phys. Rev. Lett. 77, 175 (1996).

10. S. Yunoki and A. Moreo, Phys. Rev. B58, 6403 (1998). 
11. Simulations with other distributions of random numbers lead to similar results.

12. A. Moreo, S. Yunoki, and E. Dagotto, Phys. Rev. Lett. 83, 2773 (1999).

13. See contributions by T. Natterman (cond-mat/9705295) and D. P. Belanger (condmat/9706042) to Spin Glasses and Random Fields, ed. A. P. Young, World Scientific.

14. Y. Imry and S. K. Ma, Phys. Rev. Lett. 35, 1399 (1975). See also M. Aizenman and J. Wehr, Phys. Rev. Lett. 62, 2503 (1989).

15. T. Hotta et al., preprint.

16. J. M. De Teresa et al., Phys. Rev. B56, 3317 (1997).

17. M. Kubota et al., cond-mat/9902288.

18. S. Mori, C. H. Chen, and S.-W. Cheong, Phys. Rev. Lett. 81, 3972 (1998).

19. The results of this paper are also related to the relaxor FM picture of Cr-doped $\mathrm{Nd}_{1 / 2} \mathrm{Ca}_{1 / 2} \mathrm{MnO}_{3}$ very recently discussed in T. Kimura, Y. Tomioka, R. Kumai, Y. Okimoto, and Y. Tokura, Phys. Rev. Lett. 83, 3940 (1999).

20. G. Cao et al., Phys. Rev. B56, 5387 (1997).

21. D. D. Sarma et al., Phys. Rev. Lett. 80, 4004 (1998). These authors observed that the usual paradigms are not enough to describe $\mathrm{LaNi}_{1-\mathrm{x}} \mathrm{M}_{\mathrm{x}} \mathrm{O}_{3}(\mathrm{M}=\mathrm{Mn}, \mathrm{Fe})$, and they anticipated that disorder plays an important role in this compound.

22. S. Yoon et al., Phys. Rev. B58, 2795 (1998); J. L. Gavilano et al., Phys. Rev. Lett. 81, 5648 (1998); S. L. Cooper, private communication.

23. A. Husmann et al., Science 274, 1874 (1996).

24. The effects described in this paper are different from other proposals for manganites where disorder leads to carrier localization. In such a context the formation of giant clusters is unnatural, and first-order transitions in non-disordered models are not needed for the localization to occur. The large static clusters found here must also be distinguished from the dynamical small cluster formation at finite temperature in non-disordered models, with clusters evolving rapidly with MC time, changing shapes and sizes such that the translational invariance is restored when time-averaged (5). On the other hand, the disorder described here pins the cluster at particular locations, it involves phases with the same density, and the typical sizes are much larger than found in previous simulations (5). Finally, note that the clusters in Figs.1-3 should 
also not be confused with metastable states arising from the first-order character of the transition in non-disordered models.

25. The authors thank W. Bao, S.-W. Cheong, V. Dobrosavljević, T. Hotta, and K. Yang for very useful discussions. A.M. and E.D. are supported in part by grant NSF-DMR9814350. A.F. thanks the Fundacion Antorchas for partial support.

\section{Figure Captions}

1. (A-C) are $\mathrm{MC}$ results for the two-orbital model with $\langle\mathrm{n}\rangle=0.5, \mathrm{~T}=1 / 100, \mathrm{~J}_{\mathrm{H}}=\infty, \lambda=1.2$, $\mathrm{t}=1, \mathrm{PBC}$, and $\mathrm{L}=20$ (chain size). (A) is the energy per site vs $\mathrm{J}_{\mathrm{AF}} / \mathrm{t}$ for the nondisordered model. A level crossing (first-order transition) between FM and AF states occurs; (B) MC averaged nearest-neighbor $t_{2 g}$-spins correlations vs position along the chain for one set of random $\mathrm{t}_{\mathrm{ab}}^{\alpha}$ and $\mathrm{J}_{\mathrm{AF}}$ couplings such that $\mathrm{J}_{\mathrm{AF}} / \mathrm{t}$ at every site lies between $0.21-\delta$ and $0.21+\delta$, with $\delta=0.01$. FM and AF regions are highlighted; $(\mathrm{C})$ Same as (B) but using $\delta=0.05$; (D-F) are results for the one-orbital model with $\langle\mathrm{n}\rangle=0.5$, $\mathrm{T}=1 / 70, \mathrm{~J}_{\mathrm{H}}=\infty, \mathrm{t}=1, \mathrm{OBC}$, and $\mathrm{L}=64$. (D) is the energy per site vs $\mathrm{J}_{\mathrm{AF}}$ for the nondisordered model, showing the level crossing between $\mathrm{FM}$ and $\mathrm{AF}$ states at $\mathrm{J}_{\mathrm{AF}} \sim 0.14$; (E) are the MC averaged nearest-neighbor $t_{2 g}$-spin correlations vs position for one distribution of random hoppings and $t_{2 \mathrm{~g}}$ exchanges such that $\mathrm{J}_{\mathrm{AF}} / \mathrm{t}$ is now distributed between $0.14-\delta$ and $0.14+\delta$, with $\delta=0.01$; (F) Same as (E) but with $\delta=0.03$.

2. Results of a MC simulation of the one-orbital model with a random chemical potential, PBC, $\mathrm{J}_{\mathrm{H}}=8.0$, and $\mathrm{J}_{\mathrm{AF}}=0.0$, in units of $\mathrm{t}=1$. (A) $\langle\mathrm{n}\rangle$ vs $\mu$ for a $\mathrm{L}=20$ chain at $\mathrm{T}=1 / 75$ using 24,000 MC sweeps per $\left\{\phi_{i}\right\}$ set. The results are averages over $\sim 100$ of $\left\{\phi_{i}\right\}$ configurations for the values of $\mathrm{W}$ shown; (B) Nearest-neighbors $t_{2 \mathrm{~g}}$-spin correlations vs their location along a $\mathrm{L}=60$ chain with $\mu=-6.7$ and $\mathrm{T}=1 / 75$. Shown are results for one representative $\mathrm{MC}$ snapshot, $\mathrm{W}=0.25$ (upper panel) and $\mathrm{W}=1.0$ (lower panel). Other snapshots differ from this one only by small fluctuations. The FM-AF clusters remain pinned at the same locations as the simulation evolves; (C) Density of states at $\mathrm{T}=1 / 75, \mathrm{~L}=20$ and $\mu=-6.7$ showing the presence of a pseudogap. The average density is $\langle\mathrm{n}\rangle \sim 0.87$; (D) Results of a representative MC snapshot for an $8 \times 8$ cluster, $\mathrm{T}=1 / 50, \mu=-6.2$ (close to the critical value), and $\mathrm{W}=1.0$. Regions with FM or AF nearest-neighbor $\mathrm{t}_{2 \mathrm{~g}}$-spin correlations are shown. 
3. Results of a MC simulation of the 2D RFIM at $\mathrm{T}=0.4(\mathrm{~J}=1)$, with PBC. The dark (white) small squares represent spins up (down). At $\mathrm{T}=0.4$ the thermal fluctuations appear negligible and the results shown are those of the lowest energy configuration. (A) was obtained for $\mathrm{W}=3, \mathrm{H}_{\text {ext }}=0$ using a $100 \times 100$ cluster and one set of random fields $\left\{\mathrm{h}_{\mathbf{i}}\right\}$. Typical cluster sizes are $\sim 10 a$; (B) Same as (A) but with $\mathrm{W}=1.5$. The cluster sizes have grown to $\sim 50 a$; (C) Results using a $500 \times 500$ cluster with $\mathrm{W}=1.2, \mathrm{H}_{\text {ext }}=0$ and for one configuration of random fields. The giant and percolative-like features of the clusters are apparent in the figure; (D) Same as (C) but now contrasting results between zero and nonzero $\mathrm{H}_{\mathrm{ext}}$. The dark regions are spins up in the $\mathrm{H}_{\mathrm{ext}}=0$ case, the grey regions are spins down at zero field that have flipped to up at $\mathrm{H}_{\text {ext }}=0.16$, while the white regions have spins down with and without the field. Special places are arrow marked where narrow spin down regions have flipped linking spin up domains. In (A)-(B) and (C)-(D) the same set $\left\{\mathrm{h}_{\mathbf{i}}\right\}$ was used. 

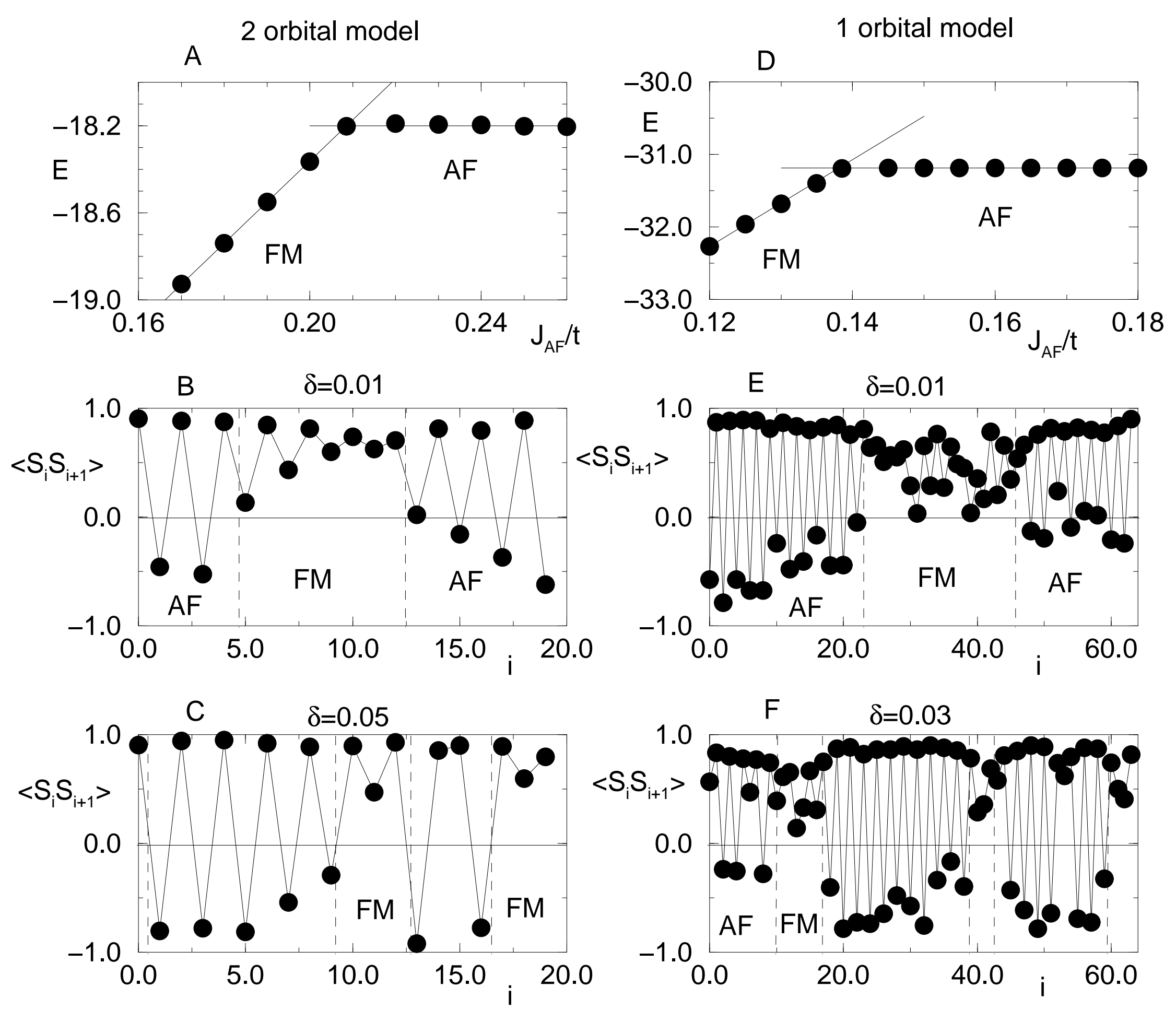

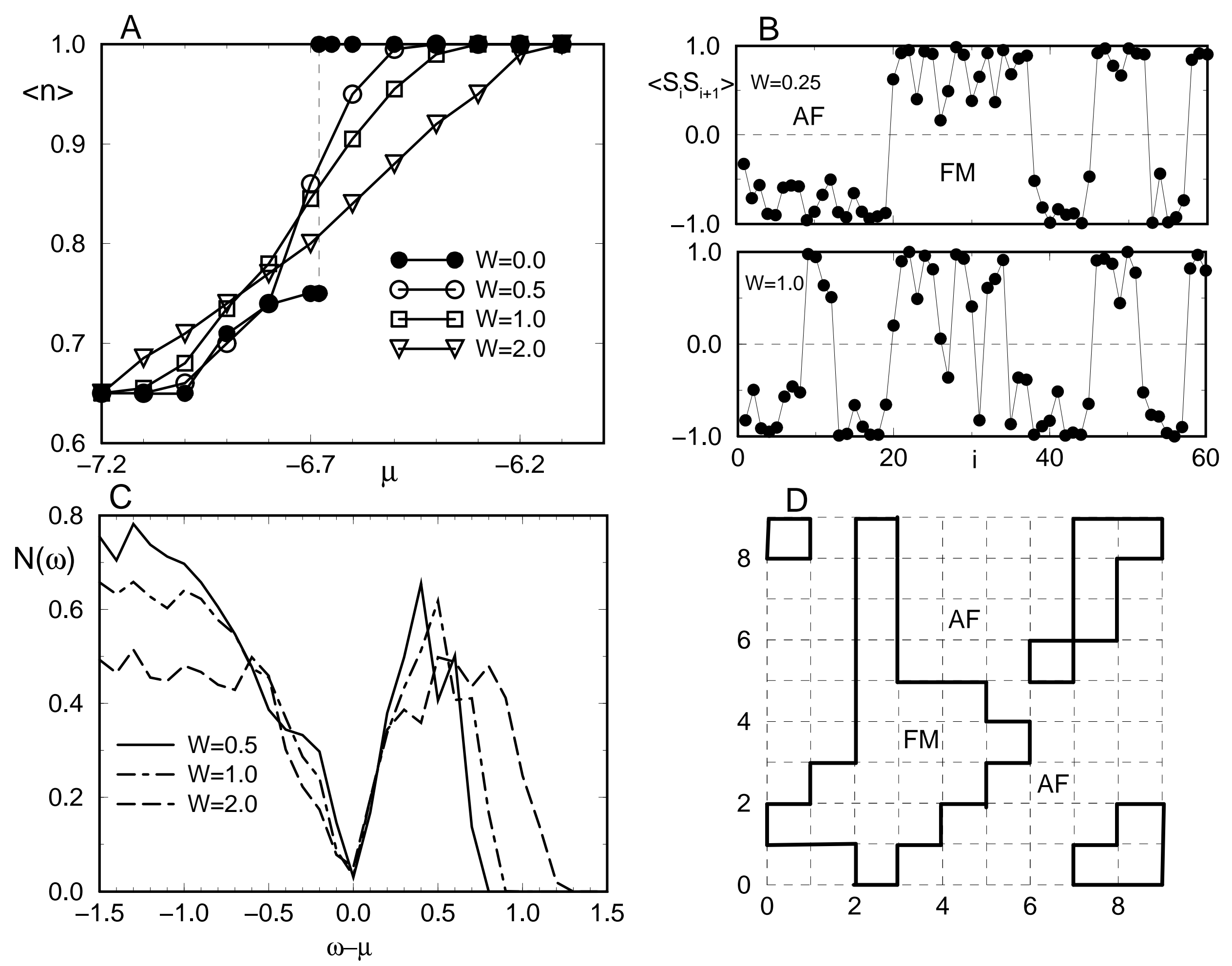


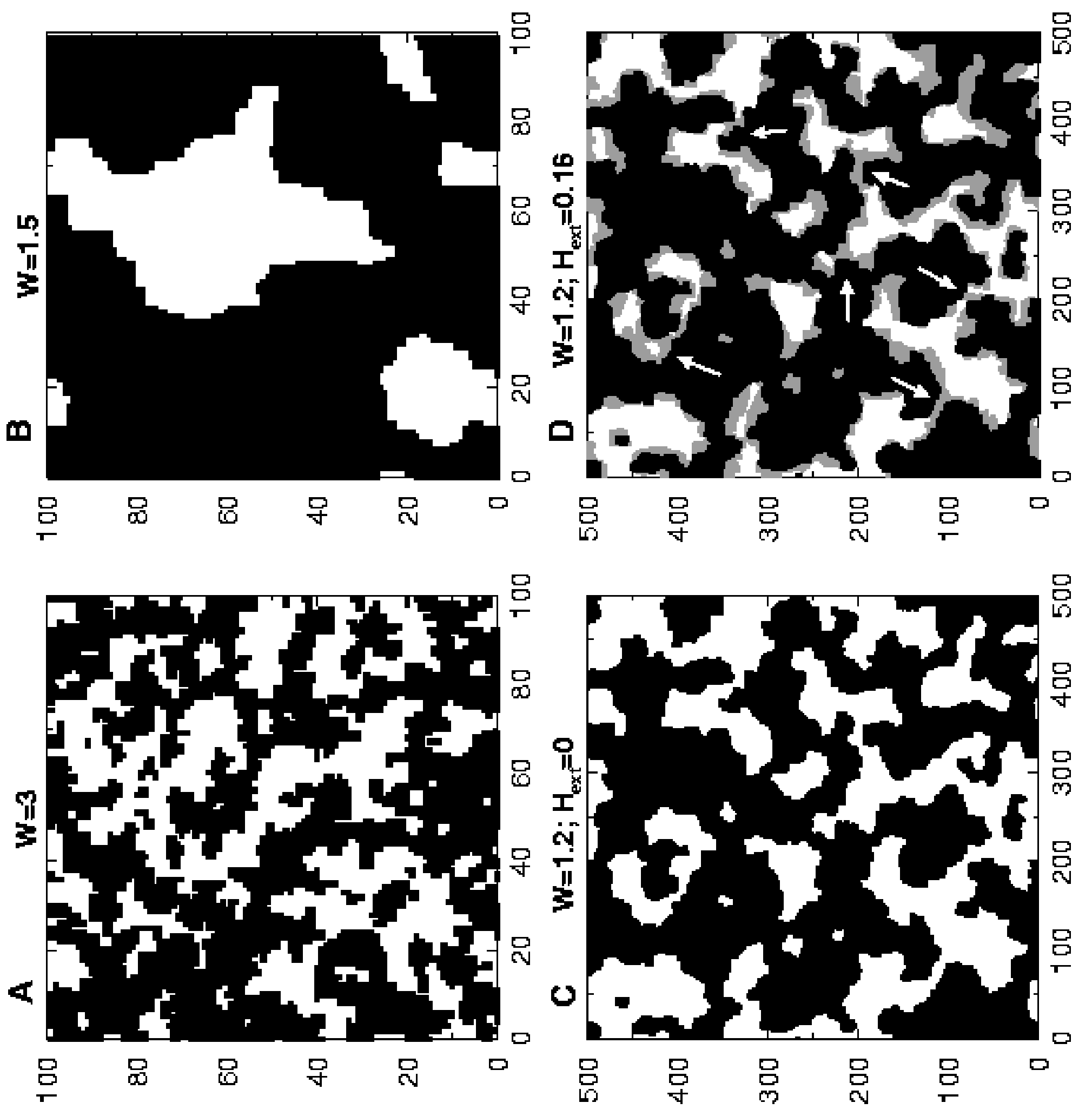

